\title{
In Situ Video Observations of the Lithiation of Single Microcrystal Graphite
}

\author{
Jared J. Lodico ${ }^{1}$, Erick Garcia ${ }^{1}$ and B. C. Regan ${ }^{1}$ \\ 1. Department of Physics \& Astronomy and California NanoSystems Institute, University of California, \\ Los Angeles, California 90095 USA
}

A graphite intercalation compound (GIC) is formed when ions or molecules (intercalant) enter between the weakly bound layers of graphite. Lithium, one of many ions that intercalates graphite, creates a highly reversible GIC that is used in commercial Li-ion batteries [1]. GICs have been studied extensively on the bulk-scale (mg) [2] and more recently on the nano-scale in an effort to understand intercalation mechanisms, electrode/electrolyte interfaces, and the failure modes of batteries [1, 2]. However, little attention has been directed towards observing the cycling process in situ for micro-scale graphite. Here, in preparation for in situ electron microscopy experiments [3, 4], we use optical microscopy to observe the lithiation and delithiation of a single microcrystal graphite flake $(0.35 \mu \mathrm{g})$ in $1 \mathrm{M}$ solution of lithium perchlorate $\left(\mathrm{LiClO}_{4}\right)$ in ethylene carbonate $(\mathrm{EC})$ and dimethyl carbonate (DMC) in a 1:1 volume ratio.

GICs are categorized by their stage number, which is the number of graphene layers between each intercalant layer. For example, in a stage 5 GIC, five graphene layers separate single intercalant layers in a repeating 5-1-5-1... pattern. Stage 1 is fully intercalated, with intercalant and graphene layers alternating. Graphite intercalated with lithium becomes blue, red, and gold at stages 3, 2 and 1 respectively [5]. These color changes roughly identify the extent to which a graphite crystal is lithiated.

We exfoliate natural graphite with adhesive tape and transfer a suitable flake to a glass slide with a prepatterned platinum electrode (Figure 1a). Each flake's thickness $(\sim 0.2-2 \mu \mathrm{m})$ and area is measured using a Bruker NT9300 Optical Profiler. The assembly of the electrochemical cell, with lithium foil reference (RE) and counter (CE) electrodes, is completed in an argon atmosphere.

Using a Gamry 600 potentiostat we cycled graphite from the open circuit potential (OCP) of $2.2 \mathrm{~V}$ to $5 \mathrm{mV}$, and then back to the OCP. Color video obtained with optical microscopy of a graphite flake undergoing such cyclic voltammetry (CV) reveals the color changes characteristic of lithiation and delithiation (Figures 1a-d). To make the changes more apparent, we construct difference images by subtracting the video's first frame from the other frames (Figures 1e-h). As more lithium is intercalated the graphite generally becomes bluer (Figures 1e-g), although the evident non-uniformity over the flake indicates that some regions are more intercalated than others.

More quantitative information can be extracted with digital analysis (Figure 1i) of the color (RGB) intensities of individual regions of interest (ROIs). The blue channel intensity of representative platinum (ROI 1) increases monotonically while the voltage decreases, indicating lithium plating. Once the voltage reverses the lithium begins to strip, although more slowly than it plated. In contrast, the blue channel intensity of representative graphite (ROI 2) shows an abrupt jump at $\sim 175$ min $(\sim 0.2 \mathrm{~V})$ which proves to be largely reversible. This color signal indicates intercalation to stage 3 . Surprisingly, although the current is still negative after Figure 1c, the ROI 2 signal indicates that the graphite is delithiating. Combining electrochemical transport measurements with simultaneous optical microscopy of 
microcrystal graphite shows promise for elucidating the physical and chemical processes underlying bulk intercalation.

\section{References:}

[1] J. -M. Tarascon and M. Armand, Nature 414 (2001), p. 359.

[2] M. S. Dresselhaus and G. Dresselhaus, Adv. Phys. 51 (2002), p. 1-186.

[3] E. R. White et al, ACS Nano 6 (2012), p. 6308.

[4] J. J. Lodico et al, Microscopy and Microanalysis 21 (Suppl 3), (2015), p. 1193.

[5] Y. Qi et al, J. Electrochem. Soc. 157 (2010), p. A741-A747.

[6] This work was supported in part by FAME, one of six centers of STARnet, a Semiconductor Research Corporation program sponsored by MARCO and DARPA.
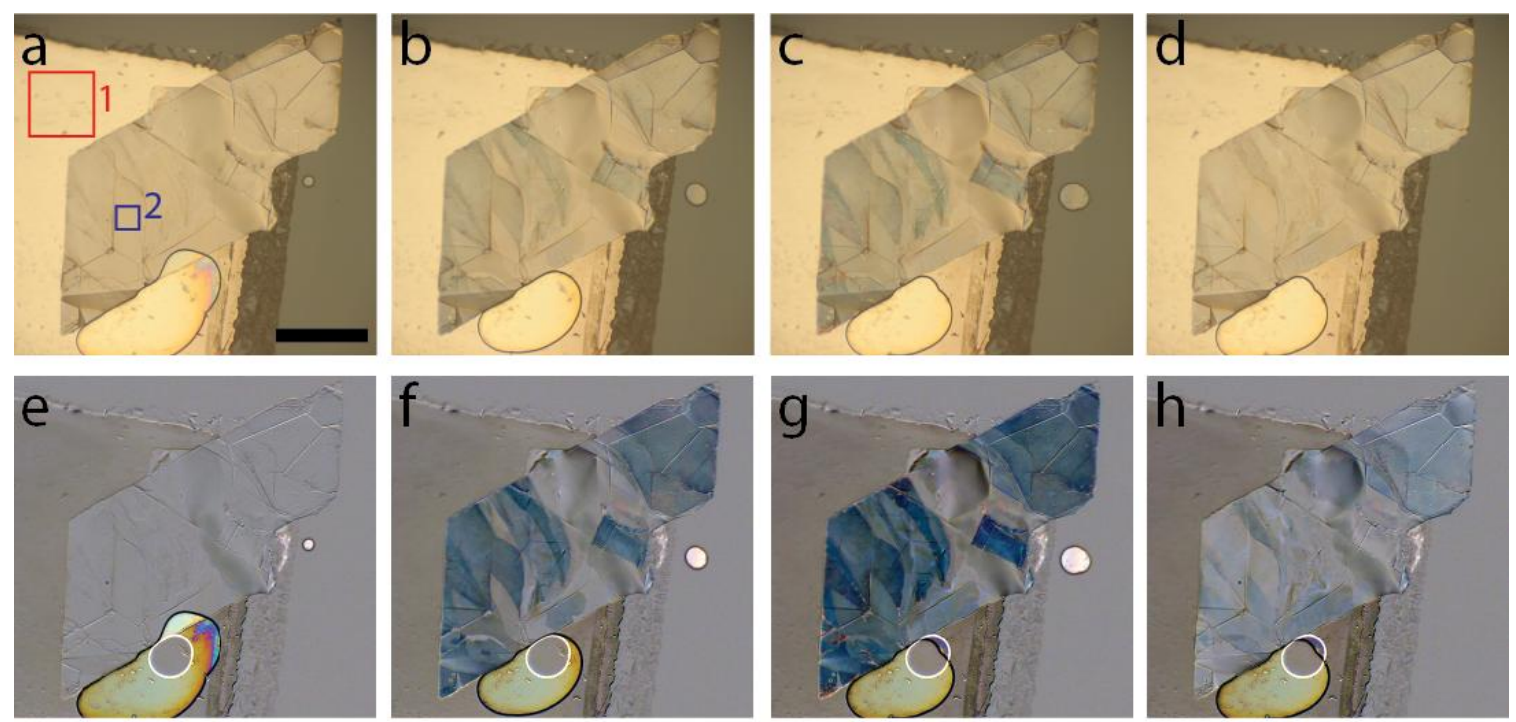

i

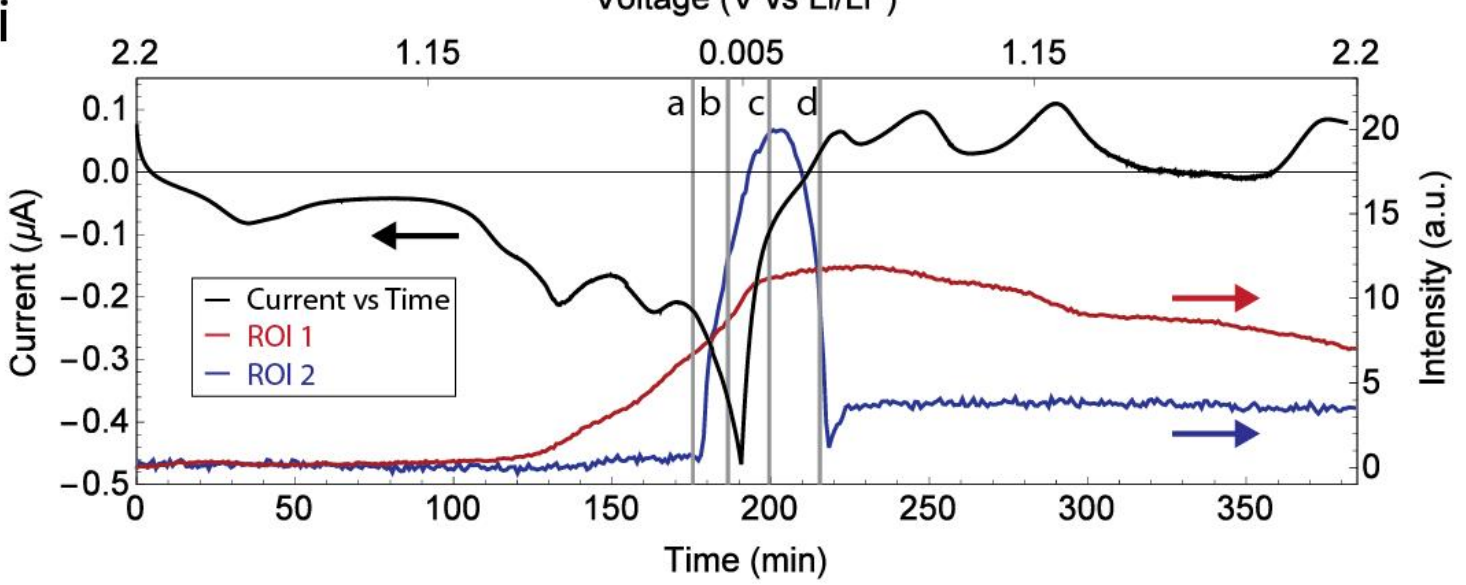

Figure 1. Raw optical images (a-d) extracted from a video show a graphite flake responding to a CV cycle. The lithium RE and CE are outside the field of view. Two rectangles in (a) designate regions of interest (ROI) for platinum (ROI 1) and graphite (ROI 2) respectively. The flake becomes bluer during lithiation (b-c), and less blue as it delithiates (d). Frames (e-h) are difference images calculated by subtracting the time $t=0 \mathrm{~s}$ frame (not shown) from (a-d). A constant voltage ramp rate of $0.2 \mathrm{mV} / \mathrm{s}$ gives the measured current and ROI average intensities which are plotted together in (i). The times corresponding to the displayed optical images are indicated by gray lines in (i). The scale bar is $200 \mu \mathrm{m}$. 